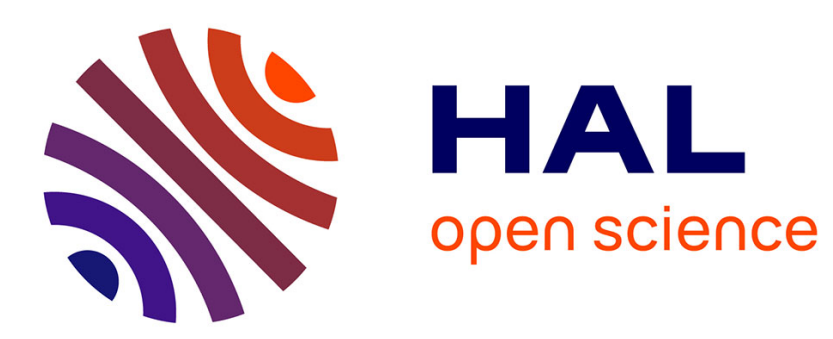

\title{
Optimization of ultrasound image quality via visual servoing
}

\author{
Pierre Chatelain, Alexandre Krupa, Nassir Navab
}

\section{To cite this version:}

Pierre Chatelain, Alexandre Krupa, Nassir Navab. Optimization of ultrasound image quality via visual servoing. IEEE Int. Conf. on Robotics and Automation, ICRA'15, May 2015, Seattle, United States. hal-01121222

\section{HAL Id: hal-01121222 \\ https://hal.inria.fr/hal-01121222}

Submitted on 27 Feb 2015

HAL is a multi-disciplinary open access archive for the deposit and dissemination of scientific research documents, whether they are published or not. The documents may come from teaching and research institutions in France or abroad, or from public or private research centers.
L'archive ouverte pluridisciplinaire HAL, est destinée au dépôt et à la diffusion de documents scientifiques de niveau recherche, publiés ou non, émanant des établissements d'enseignement et de recherche français ou étrangers, des laboratoires publics ou privés. 


\title{
Optimization of ultrasound image quality via visual servoing
}

\author{
Pierre Chatelain ${ }^{1}$, Alexandre Krupa ${ }^{2}$ and Nassir Navab ${ }^{3}$
}

\begin{abstract}
In this paper we propose a new ultrasoundbased visual servoing framework, for the optimization of the positioning of an ultrasound probe manipulated by a robotic arm, in order to improve the quality of the acquired ultrasound images. To this end, we use the recent framework of ultrasound confidence map, which aims at estimating the perpixel quality of the ultrasound signal based on a model of sound propagation in soft tissues. More specifically, we treat the ultrasound confidence maps as a new modality to design a visual servoing control law for image quality optimization. The proposed framework aims at improving ultrasound imaging techniques, such as robotic tele-echography, target tracking or volume reconstruction. Here we illustrate our approach with the application of robotic tele-echography. Experiments are performed on both an ultrasound examination training phantom and $e x$ vivo tissue samples.
\end{abstract}

\section{INTRODUCTION}

Ultrasound is a widely used modality for diagnostic and intraoperative imaging. Despite its low signal-to-noise ratio compared to other imaging techniques such as computed tomography or X-rays, ultrasound imaging is attractive because of its non-invasiveness, low cost, and high frame rate. However, the quality of ultrasound images depends on both the intrinsic properties of the device (the transducer design and various imaging parameters) and extraneous factors such as the placement of the probe, the contact force with the body, and the distribution of ultrasound gel.

The impact of the transducer design on the image quality has been extensively studied [1], [2], leading to significant improvements of the hardware over the last decades. On the other hand, a wide community of researchers has focused on the enhancement of ultrasound image quality via postprocessing algorithms such as denoising or speckle reduction [3], [4], or the use of contrast-enhanced ultrasound [5], where ultrasound contrast agents consisting of gas-filled microbubbles are injected into the circulatory system in order to increase the contrast of the ultrasound images. A review of pre- and post-processing ultrasound image enhancement techniques can be found in [6].

More recently, El-Zehiry et al. [7] have proposed an automatic optimization of the ultrasound imaging parameters to improve the quality of the images. The main advantage of this approach compared to conventional image enhancement

\footnotetext{
${ }^{1}$ Pierre Chatelain is with Université de Rennes 1, IRISA, France and Computer Aided Medical Procedures, Technische Universität München, Germany pierre.chatelaineirisa.fr

${ }^{2}$ Alexandre Krupa is with Inria Rennes - Bretagne Atlantique, IRISA, France a lexandre.krupa@inria.fr

${ }^{3}$ Nassir Navab is with Computer Aided Medical Procedures, Technische Universität München, Germany and the Johns Hopkins University, MD, USA nassir.navabein.tum.de
}

techniques is that the optimization is performed on the settings of the imaging device rather than by post-processing, therefore actually improving the quality of the raw signal.

Although the placement of the probe is known to have a significant impact on the quality of the image content (see e.g. [8]), very little work has been published on this topic. In this paper we investigate the relation between the probe positioning and the image quality, and design a new visual servoing technique to guide a probe attached to the end-effector of a robotic arm, in order to improve the image quality. The proposed approach is intended to be combined with other ultrasound-based visual servoing methods. It could for instance be used in the context of tracking, where improving the image quality would in turn ease the detection of the target.

Ultrasound-based visual servoing is a visual servo-control scheme where the control law is a function of features extracted from the ultrasound images. This framework has been used in different applications of robot-assisted ultrasound imaging. For instance, in [9], Abolmaesumi et al. control the in-plane motion of a $2 \mathrm{D}$ probe to track a section of the carotid artery. In [10] an intensity-based method is proposed to track the out-of-plane translation of the carotid artery. In [11] intensity-based visual servoing is used to compensate for organ motion. A method to maintain visibility constraints during tele-echography is proposed in [12]. The main objective of robot-assisted ultrasound imaging is to ease or automatize the manipulation of the probe, which can be exhausting during prolonged examination. In this spirit, we propose an example application of the new visual servoing method designed in this paper, where the goal is to robotically assist the operator by automating the in-plane rotation of the probe in a way that improves the image quality. To this end, our visual servoing scheme relies on ultrasound quality features.

Evaluating the quality of ultrasound image is a challenging issue, because quality is a subjective concept, and its definition can vary a lot from an observer to another, or depending on the nature of the examination. Few quality measures for ultrasound have been proposed in the literature, and most of them are based on general image analysis techniques [3], [13], [14]. In [7] the authors proposed a new quality evaluation method based on statistical learning from expert ratings. However all these methods provide only a per-image quality measure, from which it is difficult to design a control law for quality optimization. In [15] Karamalis et al. designed a new framework for ultrasound signal loss estimation, providing a per-pixel measure of the confidence in the ultrasound signal. The computation of 
Karamalis' confidence maps is based on a simplified model of sound propagation in soft tissues, and therefore provides an objective measure of quality. This framework has been successfully applied to different applications such as shadow detection and ultrasound reconstruction [15], or ultrasound image compounding [16]. Hennersperger et al. [17] have recently generalized this signal loss estimation method to arbitrarily sampled 2D and 3D data. We propose here to use the confidence maps as a new signal for servo-control, and to this end we investigate the relation between the probe motion and the variations of the confidence map.

After recalling the definition of the confidence map (section II-A), we describe in section II-B our confidence-based control law and the tele-echography framework. We present in section III the results of experiments performed on an ultrasound training phantom and ex vivo tissue samples.

\section{METHODS}

\section{A. Ultrasound Confidence maps}

In medical ultrasonography, focused ultrasound waves are generated and sent into the body by a piezoelectric transducer array. The waves propagating through the tissues undergo acoustic attenuation due to the medium viscosity and reflections at the boundaries between tissues of different densities. The echoes returning to the transducer are converted back into electric pulses and processed in order to form a $2 \mathrm{D}$ image. The resulting images have a typically low signal-tonoise ratio compared to other imaging modalities, because of the important attenuation of the ultrasound waves and the presence of artifacts due to reverberation. Therefore the quality of the signal is usually non-uniform in the image.

Ultrasound confidence maps were introduced by Karamalis et al. [15] as a per-pixel measure of the confidence for ultrasound images. This signal loss estimation method relies on a graphical representation of the image, where the nodes represent the pixels, and the edges model the sound propagation constraints (attenuation, reflection, refraction). The graph neighborhood can be either 4- or 8-connected. In our setup we consider a 4-connected graph. Indeed, the computation of the confidence map on a 4-connected graph provides a precision which is sufficient for our application, while complying with the real-time constraint. The computation with a 8-connected graph is more time-consuming and provides no noticeable improvement on the servoing behavior for the considered application.

Karamalis' confidence is defined as the probability of a random walk starting from a pixel to reach each of the virtual transducer elements under ultrasound-specific constraints. Thus it provides a per-pixel measure of uncertainty of the ultrasound signal, based on a model of sound wave propagation in soft tissues.

Given an ultrasound image $I: \Omega \longrightarrow \mathbb{R}$ defined over the field of view $\Omega$, the confidence map $C: \Omega \longrightarrow[0,1]$ provides for each pixel $(x, y) \in \Omega$ the confidence $C(x, y)$ related to the signal component $I(x, y)$ (a confidence of 1 corresponds to a perfect signal confidence). In practice, it is computed using the random walks algorithm [18], with three constraints:

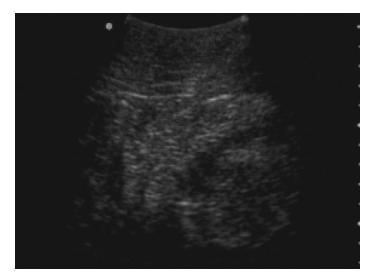

(a)

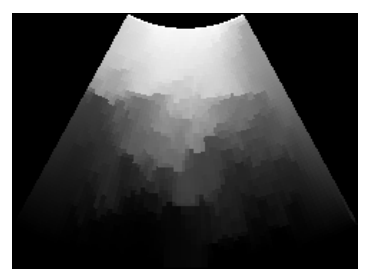

(b)
Fig. 1. (a) Ultrasound image of an ex vivo porcine tissue sample and (b) the corresponding confidence map.

- The confidence at the top of the image (next to the transducer) is 1 .

- The confidence at the bottom of the image is 0 .

- The signal propagation obeys ultrasound-specific constraints. The reader may refer to [15] for the details of the propagation model.

We show in Fig. 1 an example of confidence map computed from an ultrasound image acquired on an ex vivo porcine tissue sample.

\section{B. Control law}

We propose to use the confidence map as an additional modality to design a visual servoing framework for optimizing the quality of ultrasound imaging. We consider a convex ultrasound probe attached to the end-effector of a 6-DOF robotic arm. The field of view of the convex probe can be defined in polar coordinates as $\Omega=\left[r_{\min }, r_{\max }\right] \times\left[\theta_{\min }, \theta_{\max }\right]$, where $r_{\min }$ is the probe radius, $r_{\max }$ is the imaging depth, and $\left[\theta_{\min }, \theta_{\max }\right]$ is the imaging sector. The confidence map $C$ is computed in real-time using the method described in [15]. Our goal is to maximize the overall confidence in the image during teleoperation.

To this end, we control the in-plane rotation of the probe automatically based on the confidence map (section II-B.1). For security reasons and to maintain contact with the body, we use in addition a force control scheme (section II-B.2) for the longitudinal translation (in the direction of the image depth). The remaining 4 degrees of freedom are teleoperated by the user from a control station.

The different Cartesian frames used in our framework are represented in Fig. 2. The force sensor frame $\mathscr{F}_{s}$ is attached to a 6 -axes force/torque sensor, the probe frame $\mathscr{F}_{p}$ to the imaging center of the convex probe, and the probe contact frame $\mathscr{F}_{p c}$ to the contact point between the probe and the object's surface. These three frames are aligned along the $y$-axis of the probe. The probe gravity frame $\mathscr{F}_{g}$ is centered on the probe's mass center and its $z$-axis is aligned with the direction of the gravity force.

To design our control law we refer to the visual servoing framework [19], where the control screw $\mathbf{v}$ of the visual sensor is computed from a visual feature vector $\mathbf{s}$ as:

$$
\mathbf{v}=-\lambda \widehat{\mathbf{L}}_{s}^{+}\left(\mathbf{s}-\mathbf{s}^{*}\right)
$$

where $\lambda>0$ is the control gain, $\mathbf{s}^{*}$ is the desired value of the feature vector, and $\widehat{\mathbf{L}}_{s}^{+}$is the pseudo-inverse of 


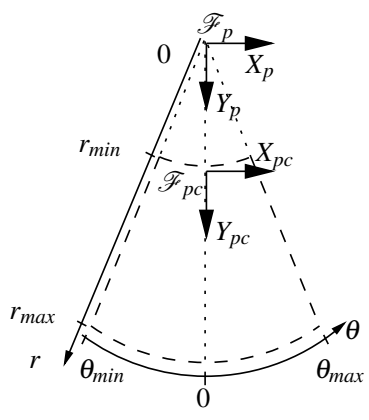

(a)

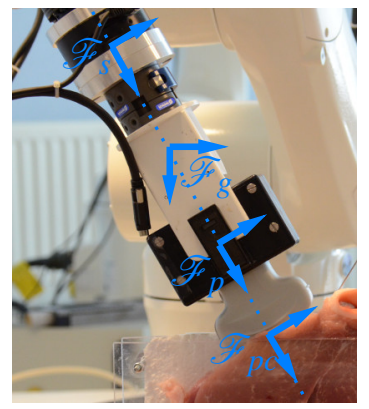

(b)
Fig. 2. Illustration of the different coordinate systems. (a) Probe frame and image polar coordinates. (b) The Viper s650 robot's end-effector and the corresponding force sensor frame $\left(\mathscr{F}_{s}\right)$, probe frame $\left(\mathscr{F}_{p}\right)$, probe contact frame $\left(\mathscr{F}_{p c}\right)$, and gravity frame $\left(\mathscr{F}_{g}\right)$.

an approximation of the interaction matrix $\mathbf{L}_{s}$ relating the variation of the features to the velocity screw, such as:

$$
\dot{\mathbf{s}}=\mathbf{L}_{s} \mathbf{v}
$$

1) Confidence control: We propose to define the feature for the confidence-based control law as the angular coordinate of the confidence-weighted barycenter of the image. Considering the confidence map $C$ defined over the polar space $\Omega=\left[r_{\text {min }}, r_{\text {max }}\right] \times\left[\theta_{\text {min }}, \theta_{\text {max }}\right]$ (see Fig. 2(a)), this feature can be computed from the image moments as:

$$
s_{c}=\frac{1}{\bar{C}} \iint_{(\theta, r) \in \Omega} \theta C(r, \theta) r d r d \theta
$$

where $\bar{C}=\iint_{(\theta, r) \in \Omega} C(r, \theta) r d r d \theta$ is the mean confidence of the current image, and $\theta=0^{\circ}$ corresponds to the central scan-line in the image.

The geometric feature defined in (3) is used to compute the in-plane angular velocity $\omega_{Z_{p}}$ to apply to the probe. In our setup, this angular velocity corresponds to the sixth component of the velocity screw in the probe frame $\mathscr{F}_{p}$ : $\mathbf{v}_{c}=\left[\begin{array}{llllll}0 & 0 & 0 & 0 & 0 & \omega_{Z_{p}}\end{array}\right]^{\top}$ (see Fig. 2). As we choose to define the control law in the probe frame, the polar image coordinate $\theta$ corresponds to the rotation around $Z_{p}$, so that we can assume the interaction matrix to be equal to the identity. Thus we can write the confidence control law as:

$$
\omega_{Z_{p}}=-\lambda_{c} a_{c}(\bar{C})\left(s_{c}-s_{c}^{*}\right)
$$

where $\lambda_{c}>0$ is the confidence control gain, $a_{c}: \mathbb{R} \longrightarrow[0,1]$ is an activation function, and $s_{c}^{*}=0^{\circ}$ is the desired value of the confidence-weighted angular feature. As $\theta=0^{\circ}$ corresponds to the central scan-line, the effect of choosing $s_{c}^{*}=0^{\circ}$ is intuitively to balance the distribution of the confidence in the field of view, bringing high-confidence areas to the center of the image.

The behavior induced by this control law is twofold:

- While scanning an homogeneous region, the probe will be oriented such as the contact along the transducer is as uniform as possible (see Fig. 3). As a result, the probe will remain orthogonal to the object's surface during the navigation.

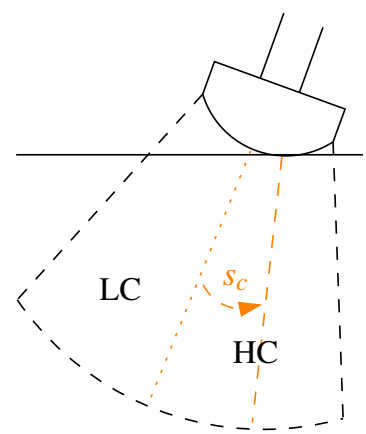

(a)

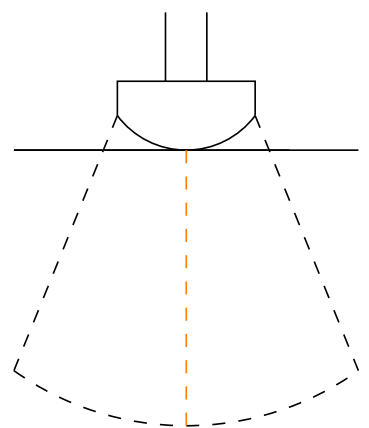

(b)
Fig. 3. Illustration of the system's behavior without obstacle. (a) Initial state: the probe orientation induces a low confidence (LC) in the left part of the image due to weaker contact force, while the right part has a high confidence (HC). (b) Final state: the probe is orthogonal to the object's surface, so that the confidence is balanced in the image.

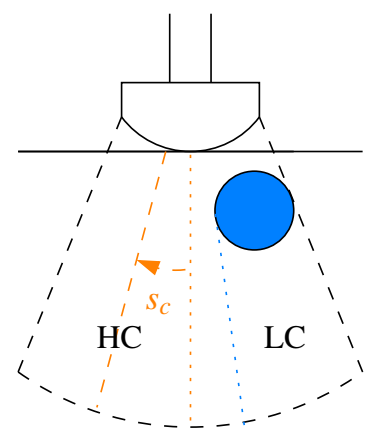

(a)

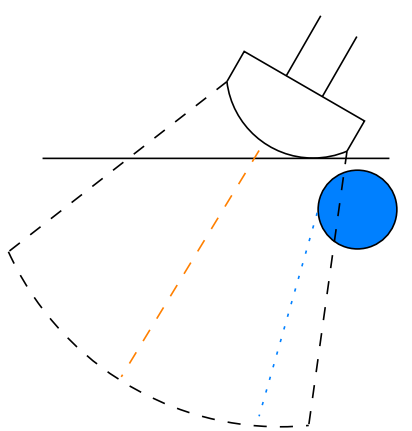

(b)
Fig. 4. Illustration of the system's behavior with obstacle. (a) Initial state: the shadowing object induces a low confidence in the right part (LC). The confidence in the left part (HC) is comparatively higher. (b) Final state: the probe is oriented such as the ultrasound beam avoids the obstacle.

- While scanning a region which contains a strongly shadowing object, such as a bone, the probe will be oriented in a way that the ultrasound beam does not go through this object (see Fig. 4). This provides a kind of obstacle-avoidance behavior during the navigation.

Note that the feature $s_{c}$ is only consistent when a sufficient contact force is applied, i.e. when the mean confidence is high enough. Therefore we use the activation function $a_{c}$ to apply the confidence control only when the mean confidence is above a certain threshold. In order to ensure smoothness of the control, we use a sigmoidal activation function to activate progressively the confidence control when the mean confidence is between $c^{-}$and $c^{+}$:

$$
a_{c}(c)= \begin{cases}0 & \text { if } c \leq c^{-} \\ \frac{1}{1+\exp \left(\frac{c^{+}-c^{-}}{c-c^{-}}-\frac{c^{+}-c^{-}}{c^{+}-c}\right)} & \text { if } c^{-}<c<c^{+} \\ 1 & \text { if } c \geq c^{+}\end{cases}
$$

2) Force control: In addition to the confidence control, we apply a force control law in order to guarantee a constant contact force between the probe and the object being scanned. We recall here briefly the force control scheme as described in [11]. 
The robot is equipped with a 6-axes force/torque sensor providing a force tensor measurement ${ }^{s} \mathbf{H}_{s}$, expressed in the sensor frame $\mathscr{F}_{s}$. We consider the transformation matrices ${ }^{p c} \mathbf{F}_{s}$ and ${ }^{s} \mathbf{F}_{g}$, which allow expressing in the frame $\mathscr{F}_{p c}$ (resp. $\mathscr{F}_{s}$ ) a force tensor known in the frame $\mathscr{F}_{s}$ (resp. $\mathscr{F}_{g}$ ). These transformation matrices depend on the relative position of the different frames, and are estimated experimentally. The gravity force tensor ${ }^{g} \mathbf{H}_{g}$ is expressed in the gravity frame $\mathscr{F}_{g}$ as ${ }^{g} \mathbf{H}_{g}=\left[\begin{array}{llllllll}0 & 0 & 9.81 m_{p} & 0 & 0 & 0\end{array}\right]^{\top}$, where $m_{p}$ is the mass of the probe. Knowing these calibration parameters, the force tensor ${ }^{p c} \mathbf{H}_{p c}$ in the contact frame $\mathscr{F}_{p c}$ can be computed as:

$$
{ }^{p c} \mathbf{H}_{p c}={ }^{p c} \mathbf{F}_{s}\left({ }^{s} \mathbf{H}_{s}-{ }^{s} \mathbf{F}_{g}{ }^{g} \mathbf{H}_{g}\right)
$$

As we aim to apply the force control only along the $y$ axis of the probe frame, this force tensor is multiplied by a selection matrix $\mathbf{M}_{s}=\operatorname{diag}(0,1,0,0,0,0)$, and the desired contact force is defined as ${ }^{p c} \mathbf{H}_{p c}^{*}=\left[\begin{array}{llllll}0 & f_{y}^{*} & 0 & 0 & 0 & 0\end{array}\right]^{\top}$ The resulting control law is defined in the probe frame as:

$$
\mathbf{v}_{s}=-\lambda_{s} \frac{\mathbf{M}_{s}{ }^{p c} \mathbf{H}_{p c}-{ }^{p c} \mathbf{H}_{p c}^{*}}{k}
$$

where $k$ is an estimation of the contact stiffness, and $\lambda_{s}$ is the force control gain.

3) Control fusion: Finally, the teleoperator controls the remaining 4 degrees of freedom (DOF) of the robotic system. The teleoperation control outputs $v_{X_{p}}, v_{Z_{p}}, \omega_{X_{p}}$ and $\omega_{Y_{p}}$ sent by the user are expressed in the probe frame $\mathscr{F}_{p}$, so that the operator controls the lateral translation and the out-of-plane motion of the probe:

$$
\mathbf{v}_{o p}=\left[\begin{array}{llllll}
v_{X_{p}} & 0 & v_{Z_{p}} & \omega_{X_{p}} & \omega_{Y_{p}} & 0
\end{array}\right]^{\top}
$$

This teleoperation control is combined with the confidence and force controls, yielding the following control law for robot's end-effector:

$$
\mathbf{v}_{e}={ }^{e} \mathbf{V}_{p}\left(\mathbf{v}_{s}+\mathbf{v}_{c}+\mathbf{v}_{o p}\right)
$$

where ${ }^{e} \mathbf{V}_{p}$ is the velocity transformation matrix from the probe frame $\mathscr{F}_{p}$ to the end-effector frame $\mathscr{F}_{e}$. Note that the force, confidence and manual controls are decoupled, since they are performed on different axes of the probe frame.

\section{RESULTS}

\section{A. Experimental setup}

For all experiments we used a Viper s650 robot (Adept Technology Inc., USA), equipped with a 6-axes force/torque sensor and a SonoSite C60 5-2 MHz convex ultrasound probe (FUJIFILM SonoSite Inc., USA) rigidly attached to its end-effector. The ultrasound frames were grabbed from the SonoSite 180PLUS ultrasound system and processed on a workstation (Intel Xeon CPU @2.1 GHz), which provided user interaction for the teleoperation, and communication with the robot. The images were grabbed at a frequency of $25 \mathrm{~Hz}$ and processed in less than $40 \mathrm{~ms}$. While the confidence and teleoperation control velocities $\mathbf{v}_{c}$ and $\mathbf{v}_{o p}$ were updated every $40 \mathrm{~ms}$, the force control was performed with a higher frequency $(200 \mathrm{~Hz})$, and the control law (9) was updated with a period of $5 \mathrm{~ms}$. The experiments were performed on

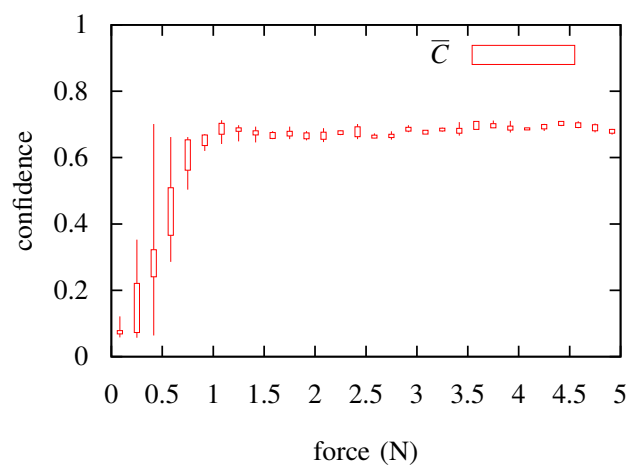

Fig. 5. Relation between the mean image confidence and the contact force. The confidence measures are grouped in force bins spaced of $0.16 \mathrm{~N}$. The boxes represent the first and last quartiles of the measured mean confidence for each force bin, while the whiskers represent the minimum and maximum values. Each bin contains approximately 20 measures of confidence.

an ABDFAN ultrasound examination training model (Kyoto Kagaku Co., Japan) simulating a patient's abdomen, and on ex vivo porcine samples.

\section{B. Confidence vs. contact force}

In our first experiment, we studied the variation of the mean confidence value with the force contact. Although this experiment is not directly related to our control framework, we include it here as we believe it provides a first insight on the dynamic behavior of the confidence maps, and validates its use as the measure of quality. The probe was progressively put in contact with the phantom, the contact force varying from $0 \mathrm{~N}$ (no contact) to $5 \mathrm{~N}$. The corresponding confidence values are displayed in Fig. 5. We observe that the mean confidence value increases rapidly while contact is made (between 0 and $1 \mathrm{~N}$ ), and then varies only slightly. This is consistent with empirical observation, as we know that a sufficient contact force is necessary to obtain a good quality image.

\section{Convergence}

The second experiment, performed on the ABDFAN phantom, demonstrates the fitness of the chosen feature. The probe was initially positioned above the phantom, without contact, and inclined with respect to the phantom's surface with an angle of approximately $10^{\circ}$. Then the control law (9) was used with a desired force $f_{y}^{*}=3 \mathrm{~N}$, a desired confidencebased angular feature $s_{c}^{*}=0^{\circ}$ and no manual interaction on the 4 teleoperated DOF, so that the probe was progressively put in contact with the phantom and converged towards an optimal placement. The control gains where set empirically to $\frac{\lambda_{s}}{k}=0.001$ for the force control and $\lambda_{c}=0.5$ for the confidence-driven control. The activation thresholds for the confidence control, defined in (5), were set empirically to $c^{-}=0.1$ and $c^{+}=0.5$.

We present in Fig. 6(a) the evolution of the confidence feature $s_{c}$ and in Fig. 6(b) the control velocities in the probe frame. The system successfully converged, with a final feature error of $0.078^{\circ}$. The mean image confidence 


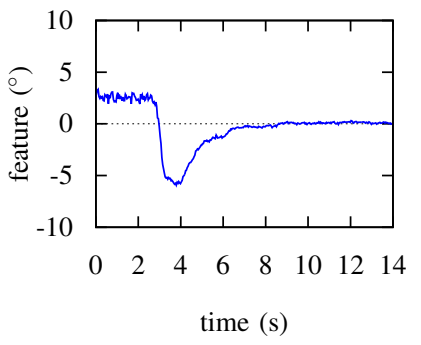

(a)

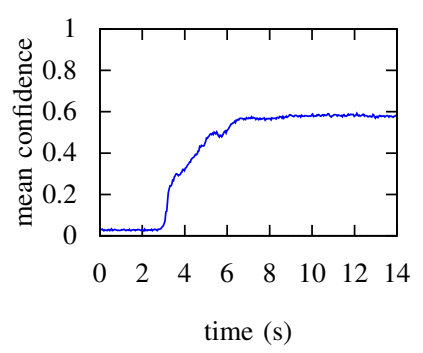

(c)

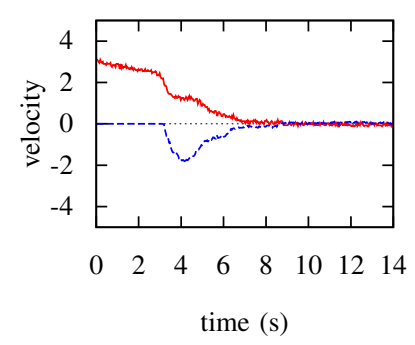

(b)

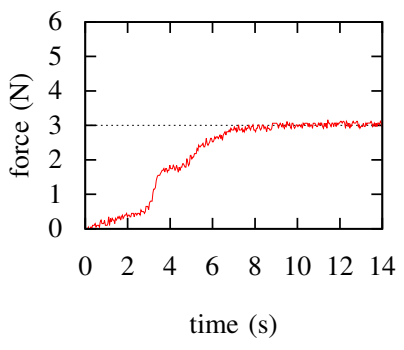

(d)

Fig. 6. Phantom experiment: Evolution of the system while establishing contact with the phantom. (a) Confidence-based feature $s_{c}$ in degrees. (b) Control velocities: $v_{Y_{p}}$ in $\mathrm{mm} / \mathrm{s}(-)$ and $\omega_{Z_{p}}$ in $\%$ s (- - ). (c) Mean image confidence $\bar{C}$. (d) Contact force $f_{y}$ in $\mathrm{N}$. The force control was activated from $t=0 \mathrm{~s}$. The confidence control started at $t=3.1 \mathrm{~s}(\bar{C}>0.1)$, and was fully activated from $t=6.0 \mathrm{~s}(\bar{C}>0.5)$ until convergence.

and the contact force value are represented in Fig. 6(c) and (d) respectively. This experiment also shows that the overall quality of the image was improved by our confidence-based servoing method, as the mean confidence increased from 0.25 (at $t=3.4 \mathrm{~s}$ ) to 0.58 (at $t=14 \mathrm{~s}$ ). The measured contact force at $t=3.4 \mathrm{~s}$ was already $1.5 \mathrm{~N}$, hence the behavior depicted in Fig. 5 suggests that this increase in confidence is mainly due to the rotation of the probe rather than to the force control.

We also performed the same experiment on an ex vivo porcine sample, in order to validate our approach on biological tissues. For this experiment the same control gains were used, but we allowed a higher force contact $f_{y}^{*}=4 \mathrm{~N}$. We also adjusted the thresholds to the tissue properties, setting $c^{-}=0.1$ and $c^{+}=0.4$. As illustrated in Fig. 7 , the system also converged successfully in this experiment, with a final feature error of $0.050^{\circ}$.

\section{Tele-echography}

Finally we illustrate here the applicability of the proposed method in the context of teleoperation. The objective is to ease the manipulation of the probe by optimizing its orientation during the teleoperated examination. The probe was controlled according to (9), so that 4 degrees of freedom were controlled manually by the operator. The desired contact force was set to $f_{y}^{*}=5 \mathrm{~N}$. During the navigation, the probe orientation adapted automatically an orientation providing a good image quality, even when scanning shadowed areas such as behind the ribs. We display in Fig. 8

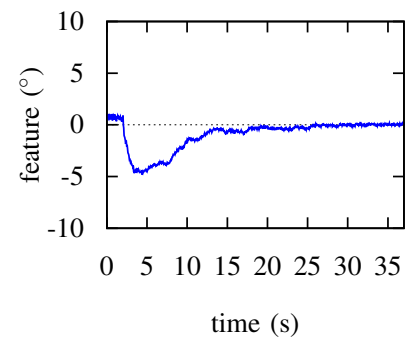

(a)

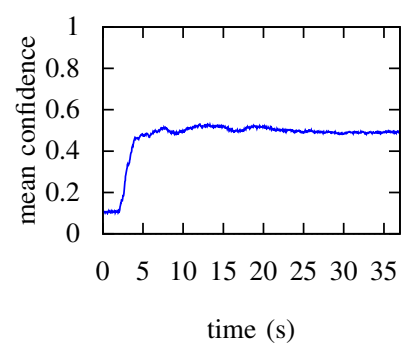

(c)

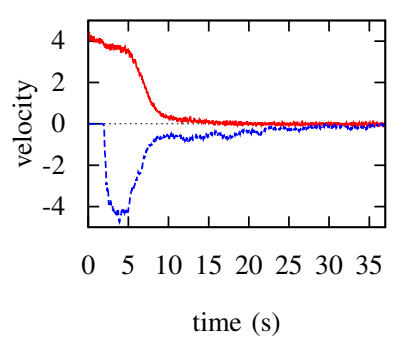

(b)

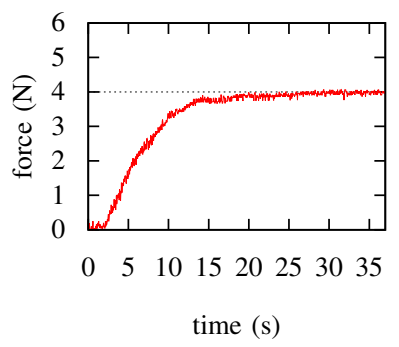

(d)
Fig. 7. Ex vivo experiment: Evolution of the system while establishing contact with the ex vivo porcine tissue sample. (a) Confidence-based feature $s_{c}$ in degrees. (b) Control velocities: $v_{Y_{p}}$ in $\mathrm{mm} / \mathrm{s}(-)$ and $\omega_{Z_{p}}$ in $\%$ s (- -). (c) Mean image confidence $\bar{C}$. (d) Contact force $f_{y}$ in $\mathrm{N}$. The force control was activated from $t=0 \mathrm{~s}$. The confidence control started at $t=2.0 \mathrm{~s}$ $(\bar{C}>0.1)$, and was fully activated from $t=3.5 \mathrm{~s}(\bar{C}>0.4)$ until convergence.

the evolution of system (confidence feature value, velocity, mean confidence and contact force) during a selected period of a teleoperation experiment. We invite the reader to refer to the corresponding video supplied in supplementary material for a full demonstration of the method. A selection of frames extracted from this video is reproduced in Fig. 9. Between the frames presented in Fig. 9(c) and (d), the probe is guided by the operator towards the side of the phantom, over the ribs. The peak in feature error observed around $t=40 \mathrm{~s}$ in Fig. 8(a) corresponds to the instant when the probe reaches the rib cage. A peak in the contact force, due to the salient ribs, occurs at the same instant. The feature error then quickly decreases thanks to our confidence-based control scheme. As a result, although the ribs are strongly shadowing, our confidence-based visual servoing method allows the probe to be automatically re-oriented in a way that maintains the visibility of the inner tissues.

\section{CONCLUSION}

We proposed a new approach for optimizing the quality of robot-assisted ultrasound imaging, based on confidencedriven visual servoing. Although we presented here an application to tele-echography, our method is also intended to be integrated into other ultrasound-based visual servoing frameworks, in order to improve the efficiency of servoing tasks. Potential applications are for example tracking a soft tissue target, or reconstructing a 3D region from 2D frames, where our method could be used as a secondary task to 


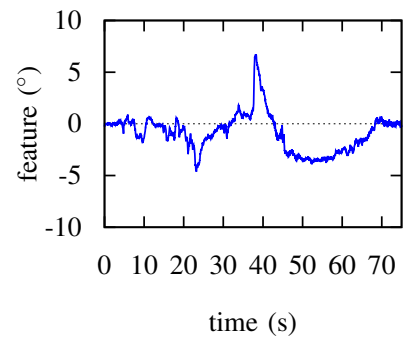

(a)

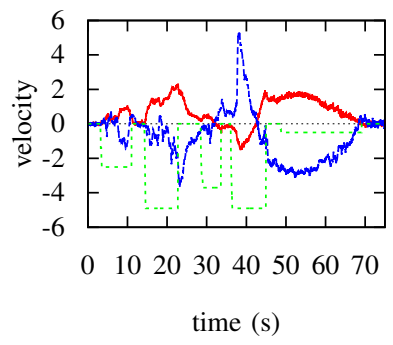

(b)

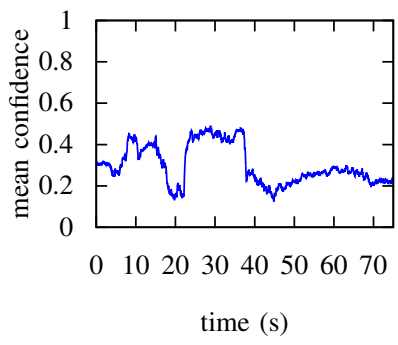

(c)

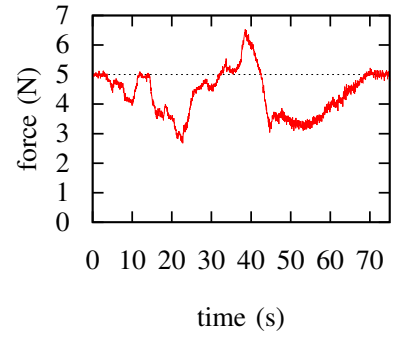

(d)

Fig. 8. Teleoperation experiment: (a) Confidence-based feature $s_{c}$ in degrees. (b) Evolution of the control velocity $v_{Y_{p}}$ in $\mathrm{mm} / \mathrm{s}(-), v_{X_{p}}$ in $\mathrm{mm} / \mathrm{s}(\cdots)$ and $\omega_{Z_{p}}$ in $\%$ s (- - ). (c) Mean image confidence $\bar{C}$. (d) Contact force $f_{y}$ in N.

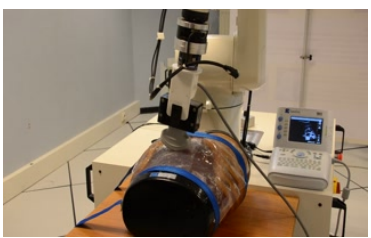

(a)

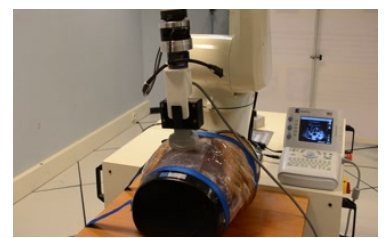

(b)

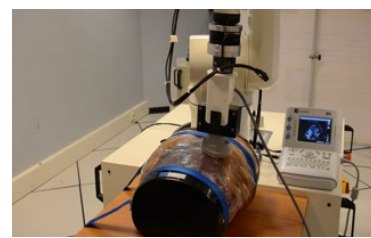

(c)

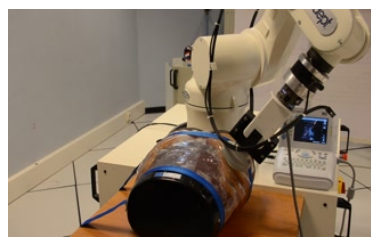

(d)

Fig. 9. Pictures of the robotic setup during a demonstration of teleoperation on an ABDFAN phantom. (a) Initial contact with the phantom. (b) Position after convergence of the confidence-based feature. (c) The operator moves the probe towards the ribs $(t=34 \mathrm{~s})$. (d) The probe is placed between the ribs $(t=74 \mathrm{~s})$

improve the image quality.

In this article we considered the control of the in-plane rotation. The results show that the confidence map can be used in control tasks to improve the image quality. In future work we will consider the confidence-driven servoing of other degrees of freedom, in particular out-of-plane motions, which could provide finer control of the image quality.

\section{ACKNOWLEDGMENT}

This work is partially supported by the BFS funding for project RoBildOR.

\section{REFERENCES}

[1] R. L. Goldberg and S. W. Smith, "Multilayer piezoelectric ceramics for two-dimensional array transducers," IEEE Trans. Ultrason., Ferroelect., Freq. Contr., vol. 41, no. 5, pp. 761-771, 1994.

[2] M. Kofler, A. Beham, M. Affenzeller, and S. Wagner, "Optimization of medical ultrasound transducers with simulation and genetic algorithms," in European Modeling and Simulation Symp., 2008, pp. 100105.

[3] C. P. Loizou, C. S. Pattichis, M. Pantziaris, T. Tyllis, and A. Nicolaides, "Quality evaluation of ultrasound imaging in the carotid artery based on normalization and speckle reduction filtering," Medical and Biological Eng. and Computing, vol. 44, no. 5, pp. 414-426, 2006.

[4] S. Tsantis, S. Spiliopoulos, A. Skouroliakou, D. Karnabatidis, J. D. Hazle, and G. C. Kagadis, "Multiresolution edge detection using enhanced fuzzy c-means clustering for ultrasound image speckle reduction," Medical physics, vol. 41, no. 7, p. 072903, 2014.

[5] B. B. Goldberg, J.-B. Liu, and F. Forsberg, "Ultrasound contrast agents: A review," Ultrasound in Medicine and Biology, vol. 20, no. 4, pp. 319-333, 1994.

[6] S. H. C. Ortiz, T. Chiu, and M. D. Fox, "Ultrasound image enhancement: A review," Biomed. Signal Process. and Control, vol. 7, no. 5, pp. 419-428, 2012.

[7] N. El-Zehiry, M. Yan, S. Good, T. Fang, S. K. Zhou, and L. Grady, "Learning the manifold of quality ultrasound acquisition," in Medical Image Computing and Computer-Assisted Intervention, Sept. 2013, pp. 122-130.
[8] M. Klimstra, J. Dowling, J. L. Durkin, and M. MacDonald, "The effect of ultrasound probe orientation on muscle architecture measurement," J. Electromyography and Kinesiology, vol. 17, no. 4, pp. 504-514, 2007

[9] P. Abolmaesumi, S. Salcudean, and W. Zhu, "Visual servoing for robot-assisted diagnostic ultrasound," in IEEE Int. Conf. Eng. Med. and Biol. Soc., vol. 4, 2000, pp. 2532-2535.

[10] R. Nakadate, J. Solis, A. Takanishi, E. Minagawa, M. Sugawara, and K. Niki, "Out-of-plane visual servoing method for tracking the carotid artery with a robot-assisted ultrasound diagnostic system," in IEEE Int. Conf. Robot. Automat., 2011, pp. 5267-5272.

[11] C. Nadeau and A. Krupa, "Intensity-based ultrasound visual servoing: modeling and validation with 2D and 3D probes," IEEE Trans. Robot., vol. 29, no. 4, pp. 1003-1015, August 2013.

[12] A. Krupa, D. Folio, C. Novales, P. Vieyres, and T. Li, "Robotized teleechography: an assisting visibility tool to support expert diagnostic," IEEE Syst. J., 2014.

[13] D. Kashyap Swathi Rangaraju, K. Kumar, and C. Renumadhavi, "Review paper on quantitative image quality assessment - medical ultrasound images," in Int. J. Eng. Research and Technology, vol. 1, no. 4, June 2012.

[14] X. Zhao, J. Zhou, W.-F. Loke, M. Chirala, and C. Zhang, "Quality evaluation of ultrasound imaging using a MATLAB test-bench," in SPIE Medical Imaging, 2013, pp. 867516-867516.

[15] A. Karamalis, W. Wein, T. Klein, and N. Navab, "Ultrasound confidence maps using random walks," Medical image analysis, vol. 16 , no. 6, pp. 1101-1112, 2012

[16] C. S. zu Berge, A. Kapoor, and N. Navab, "Orientation-driven ultrasound compounding using uncertainty information," in Information Processing in Computer-Assisted Interventions, 2014, pp. 236-245.

[17] C. Hennersperger, D. Mateus, M. Baust, and N. Navab, "A quadratic energy minimization framework for signal loss estimation from arbitrarily sampled ultrasound data," in Medical Image Computing and Computer-Assisted Intervention, Sept. 2014, pp. 373-380.

[18] L. Grady, "Random walks for image segmentation," IEEE Trans. Pattern Anal. Machine Intell., vol. 28, no. 11, pp. 1768-1783, 2006.

[19] F. Chaumette and S. Hutchinson, "Visual servo control, part I. Basic approaches," IEEE Robot. Automat. Mag., vol. 13, no. 4, pp. 82-90, 2006. 